\title{
Lightning electromagnetic pulse magnetic field calculation
}

\author{
Wei He ${ }^{1, a,{ }^{*}}$, Huafu $\mathrm{Li}^{1, \mathrm{~b}}$, Limin Feng ${ }^{1}$, Xingtuan $\mathrm{Yang}^{1}$, Qiqi He ${ }^{2}$, Jinji Zheng ${ }^{1}$, \\ Tianyi Jiang ${ }^{1}$ \\ ${ }^{1}$ School of Physics and Electronic Information, Yunnan Normal University, Kunming, China \\ ${ }^{2}$ School of Computer and Information, Yunnan University, Kunming, China \\ *Corresponding author: he99wei@aliyun.com
}

Keywords: Lightning; Electromagnetic fields; Matlab

\begin{abstract}
Lightning electromagnetic pulse is strong electromagnetic interference sources, along with the rapid development of information technology, it has all kinds of hazards of electrical and electronic equipment, communications equipment becoming more serious; interference with normal radio communications and a variety of remote control devices, and become radio an important source of noise; secondly, it is also harmful to the area around the lightning channel, and in the case of a lightning strike electrical equipment may affect the normal operation of power systems. To protect sensitive equipment from the dangers of lightning electromagnetic pulse field and select the appropriate protective measures, the paper analyzes the situation of the surrounding space radiation when lightning electromagnetic pulse propagation, by solving the Maxwell equations, using Matlab to draw the amount of space each lightning electromagnetic field Distribution. Distribution by ground lightning electromagnetic pulse in the earth study law, can get a variety of electrical parameters conclude the lightning current, lightning charge moment and charge distribution in the cloud, etc..They can provide a viable reference for positioning data and lightning warning.
\end{abstract}

\section{Introduction}

When lightning occurs will have a strong magnetic field around it and the various communication equipments caused serious interference. In lightning protection, accurate lightning electromagnetic field space distribution is essential to understand. Therefore, lightning electromagnetic field of research has important and practical significance[1,2].

Broadly speaking, all the hydrodynamic properties of the atmosphere around the mine and related thunderstorms. An important feature of thunder is hard to hear that it is accompanied by the frequency of the infrasound. Depending of the main characteristic of thunder forming process and it's propagation in the atmosphere. Thunder is the acoustic results of lightning shock wave's attenuation and the observations show that thunder of the farthest distance of $20-30 \mathrm{~km}$ which is due to atmospheric temperature with height is decreasing[3,4,5]. The thunder's propagation in the atmosphere when sound thunder bending upward. The research on the electromagnetic field of lightning began in 1975. Uman and others assumed that the earth was a perfect conductor[6]. In this case, the expression of the electromagnetic field generated by the lightning current in space is introduced. Later, in order to analysis of overhead line caused by lightning to the high voltage pulse. Master also researches and analyses the lightning generated electromagnetic field. They used the lightning current for triangle wave function or double exponential function forms to get more useful results. This paper is derived to electromagnetism is the assumption that the earth is a perfect conductor. According to the Maxwell equations that electromagnetic field in different directions of the space component analysis, expressions for the electromagnetic field distribution around the lightning current is obtained. Finally, using Matlab drawing and depicting the spatial distribution of the electromagnetic field. 


\section{Principle and calculation}

Describing the electromagnetic field on the premise is that the earth is regarded as an ideal conductor and then using the Maxwell equations[7,8]. According the distribution space to analyze and calculate. Deriving the electromagnetic characteristics of the lightning current distribution around and rendering the electromagnetic expression. Then the lightning electromagnetic field distribution of the respective amount of space was obtained using Matlab. That can see the distribution of space directly for further lightning electromagnetic field Lightning research and provide a theoretical basis. Calculation of electromagnetic fields generated by lightning also was introduced. It must be the first determine the location and route of lightning happened. It assumes that lightning occurs at the station from the communication overhead power lines are not too far away and it is linear flash. Lightning channel straight line perpendicular to the channel floor earth as an ideal conductor. As shown in Fig.1.
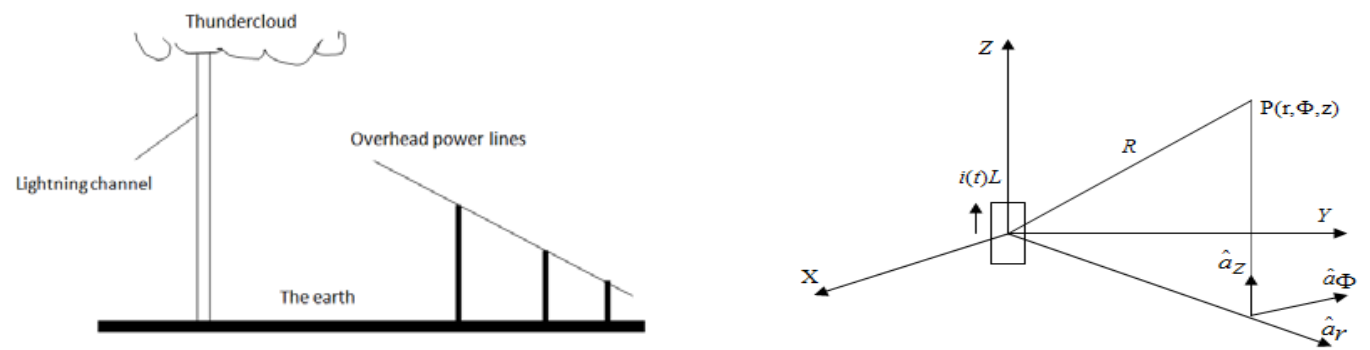

Fig. 1. (left)Lightning channel and the overhead power lines

Fig. 2. (right)A schematic view of the current element in cylindrical coordinates

If the current unit is $i\left(\vec{R}^{\prime}, t\right) L, \mathrm{~L}$ is the length of the current unit. According vector potential $\vec{A}(\vec{R}, t)$ and Maxwell equations can be obtained at any point of the electric field generated by a current element $\vec{E}(\vec{R}, t)$ and magnetic field $\vec{B}(\vec{R}, t)$ :

$$
\left\{\begin{array}{l}
\nabla \cdot \vec{E}(\vec{R}, t)=\rho\left(\vec{R}^{\prime}, t\right) / \varepsilon_{0} \\
\nabla \cdot \vec{B}(\vec{R}, t)=0 \\
\nabla \times \vec{E}(\vec{R}, t)=-\frac{\partial \vec{B}(\vec{R}, t)}{\partial t} \\
\nabla \times \vec{B}(\vec{R}, t)=\mu \vec{J}\left(\vec{R}^{\prime}, t\right)+\frac{1}{c^{2}} \frac{\partial \vec{E}(\vec{R}, t)}{\partial t}
\end{array}\right.
$$

Using vector potential $\vec{A}(\vec{R}, t)$ and Scalar potential $\Phi(\vec{R}, t)$ can be obtained Eq.2 of the Electric and the magnetic field:

$$
\vec{A}(\vec{R}, t)=\frac{\mu_{0} \vec{L}}{4 \pi}\left[i \vec{R}^{\prime}, t-\frac{\left|\vec{R}-\vec{R}^{\prime}\right|}{c}\right] /\left|\vec{R}-\vec{R}^{\prime}\right|
$$

First calculate the current element located at the origin of coordinate situation is shown in Fig.2. Since the space field generated by the lightning current column meet symmetrical features. So this article uses a cylindrical coordinate system to solve electric and magnetic fields respectively. Since $\vec{R}^{\prime}=0$ by Eq.2, we get:

$$
\vec{A}(\vec{R}, t)=\left(\frac{\mu_{0}}{4 \pi} \frac{i(0, t-R / c)}{R} L\right) \hat{a}_{z}=A_{z}(\vec{R}, t) \hat{a}_{z}
$$

Because $\vec{A}(\vec{R}, t)$ only z component, we get:

$$
\begin{aligned}
& \vec{B}(r, \Phi, z, t)=\nabla \times \vec{A}(r, \Phi, z, t)=\left(\frac{1}{r} \frac{\partial A_{z}}{\partial \Phi}-\frac{\partial A_{\Phi}}{\partial z}\right) \hat{a}_{r}+\left(\frac{\partial A_{r}}{\partial z}-\frac{\partial A_{z}}{\partial r}\right) \hat{a}_{\Phi}+\frac{1}{r}\left[\frac{\partial}{\partial r}\left(r A_{\Phi}\right)-\frac{\partial A_{r}}{\partial \Phi}\right] \hat{a}_{z} \\
& =\hat{a}_{\Phi}\left(-\frac{\partial A_{z}}{\partial r}\right)=-\frac{\mu_{0} L}{4 \pi} \frac{\partial}{\partial r}\left(\frac{i(t-R / c)}{R}\right) \hat{a}_{\Phi}
\end{aligned}
$$

We get the relationship of time and space derivative (radius) of derivation between:

$$
\frac{\partial}{\partial r}[i(t-R / c)]=-\frac{r}{c R} \frac{\partial}{\partial t}[i(t-R / c)]
$$


This is an expression requires a magnetic field, which is a direction in a plane perpendicular to the direction of the current. By the Eq. 2 would determine the expression of the electric field. According to Eq.3, we get:

$$
\nabla \cdot \vec{A}=\frac{1}{r} \frac{\partial}{\partial r}\left(r A_{r}\right)+\frac{1}{r} \frac{\partial A_{p}}{\partial \Phi}+\frac{\partial A_{z}}{\partial z}=\frac{\partial A_{z}}{\partial z}=\frac{\mu_{0} L}{4 \pi} \frac{\partial}{\partial z}\left(\frac{i(t-R / c)}{R}\right)=\frac{\mu_{0} L}{4 \pi}\left[\frac{1}{R} \frac{\partial i(t-R / c)}{\partial z}-\frac{z}{R^{3}} i(t-R / c)\right]
$$

Since the field distribution has a cylindrically symmetric property that has nothing to do with $\Phi$, therefore, the gradient operator is:

$$
\nabla=\hat{a}_{r} \frac{\partial}{\partial r}+\hat{a}_{z} \frac{\partial}{\partial z}
$$

The Eq.7 into Eq.3 in the second paragraph and get:

$$
\nabla(\nabla \cdot \vec{A})=\frac{\mu_{0} L}{4 \pi}\left[\frac{\partial}{\partial r}\left(\frac{1}{R} \frac{\partial i(t-R / c)}{\partial z}\right)-\frac{\partial}{\partial r}\left(\frac{z}{R^{3}} i(t-R / c)\right)\right] \hat{a}_{r}+\frac{\mu_{0} L}{4 \pi}\left[\frac{\partial}{\partial z}\left(\frac{1}{R} \frac{\partial i(t-R / c)}{\partial z}\right)-\frac{\partial}{\partial z}\left(\frac{z}{R^{3}} i(t-R / c)\right)\right] \hat{a}_{z}
$$

From Eq.2, we get:

$$
-\frac{\partial \vec{A}(\vec{R}, t)}{\partial t}=-\frac{L}{4 \pi \varepsilon_{0} c^{2} R} \frac{\partial i(t-R / c)}{\partial t} \hat{a}_{z}
$$

In the derivation of the above, we can see only two components of the electric field direction, in the direction $\hat{a}_{z}$ and direction $\hat{a}_{r}$ component, so that the electric field can be written as:

$\vec{E}=E_{r} \hat{a}_{r}+E_{z} \hat{a}_{z}$

The Eq.8 and Eq.9 into Eq.2, the available $E_{r}$ and $E_{z}$ components of the expression:

$$
\begin{aligned}
& E_{r}(r, \Phi, z, t)=\frac{L}{4 \pi \varepsilon_{0}}\left[\frac{3 r z}{R^{5}} \int_{0}^{t} i(\tau-R / c) d \tau+\frac{3 r z}{c R^{4}} i(t-R / c)+\frac{r z}{c^{2} R^{3}} \frac{\partial i(t-R / c)}{\partial t}\right. \\
& E_{z}(r, \Phi, z, t)=\frac{L}{4 \pi \varepsilon_{0}}\left[\frac{2 z^{2}-r^{2}}{R^{5}} \int_{0}^{t} i(\tau-R / c) d \tau+\frac{2 z^{2}-r^{2}}{c R^{4}} i(t-R / c)-\frac{r^{2}}{c^{2} R^{3}} \frac{\partial i(t-R / c)}{\partial t}\right]
\end{aligned}
$$

\section{Lightning electromagnetic fields spatial distribution}

By the expression of lightning electromagnetic:

$$
\vec{B}(r, \Phi, z, t)=\frac{\mu_{0} L}{4 \pi}\left[\frac{r}{c R^{2}} \frac{\partial i(t-R / c)}{\partial t}+\frac{r}{R^{3}} i(t-R / c)\right] \hat{a}_{\Phi}
$$

Obtain the spatial distribution of lightning electromagnetic field, the plane perpendicular to the current flow shown in Fig.3.

Fig. 3. Lightning Electromagnetic Fields Spatial Distribution

From the above discussion, only the electric field in the direction $\hat{a}_{z}$ and direction $\hat{a}_{r}$ component.Therefore, the expression by the spatial components can obtain $E_{r}$ and $E_{z}$ components of the distribution and shown in Fig.4:

$$
\begin{aligned}
& E_{r}(r, \Phi, z, t)=\frac{L}{4 \pi \varepsilon_{0}}\left[\frac{3 r z}{R^{5}} \int_{0}^{t} i(\tau-R / c) d \tau+\frac{3 r z}{c R^{4}} i(t-R / c)+\frac{r z}{c^{2} R^{3}} \frac{\partial i(t-R / c)}{\partial t}\right. \\
& E_{z}(r, \Phi, z, t)=\frac{L}{4 \pi \varepsilon_{0}}\left[\frac{2 z^{2}-r^{2}}{R^{5}} \int_{0}^{t} i(\tau-R / c) d \tau+\frac{2 z^{2}-r^{2}}{c R^{4}} i(t-R / c)-\frac{r^{2}}{c^{2} R^{3}} \frac{\partial i(t-R / c)}{\partial t}\right]
\end{aligned}
$$



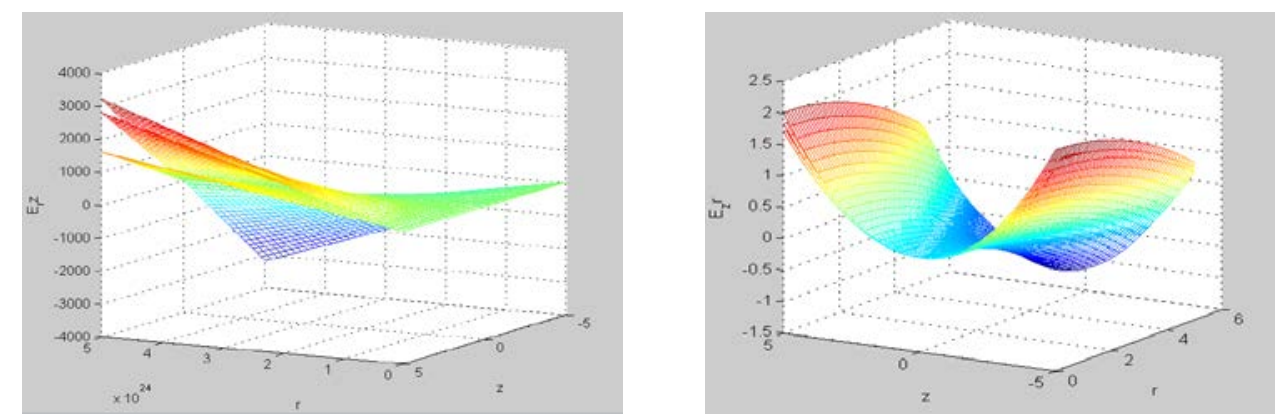

Fig. 4. (left) $E_{r}$ component and (right) $E_{z}$ component

Lightning current component generated by the lightning current and lightning current is proportional to the relationship. A lightning current derivative with respect to time and may also be seen as a lightning current steepness. It's proportional to the size and level of the ground magnetic field components. Although the ratio of the electric field induced electrostatic field spread farther but it is also relative. Induction field around the lightning current is still relatively strong. Indicating the propagation of electromagnetic radiation distance compared to the previous two are farther. Lightning produce a strong current which causes a change in the strong electromagnetic radiation and electrostatic fields. On the other hand, it can through the measured data from the lightning electromagnetic field changes obtain various electrical parameters conclude the lightning current. Lightning charge moment and charge distribution in the cloud, etc..It provides a viable reference for positioning and lightning warning. Through the study of the magnetic field around the lightning. We can provide the data about the lightning protection of the safety distance and the parameters of the lightning protection device selection, etc..

\section{Acknowledgements}

This project was supported by National Natural Science programs (51267021) and College students' innovative entrepreneurial training programs (2015).

\section{References}

[1] Feng Zhiwei, Xiao Wen, Ma Jinfu, etc. Based on the data flash to lightning current cumulative frequency formula explore [J]. Meteorology Division Technology, 2012, 40 (1): 137-140.

[2] Zhang Dianjiang. Electronic Systems Lightning electromagnetic interference and prevention [J]. Petroleum Chemical Industry, 2011, 47 (1): 68-70.

[3] Zhang Zhimin, Liu Dong. Electronic anti-lightning equipment method[J]. Modern electronic technology, 2012, 35 (21): 192-194.

[4]Zhang Qin, Feng Wanxing etc. China Power Grid lightning location system and lightning monitoring network [J]. High-voltage technology, 2008,34 (3): 425-431.

[5] M.A.Uman, D.K.McLain, E.P.Krider. The electromagnetic radiation from a finite antenna. Amer[J]. nPhys, 1975, 43 (7): 33-38.

[6] M.J.Master, M.A.Uan. Lightning induced voltages on power lines theory [J]. IEEE Trans. Power Apparatus Syst, 1984, 103 (02): 502-518.

[7] Pi-Cui Zhang, Wei He.Analysis on lightning electromagnetic fields [J].Applied Mechanics and Materials, 2013, 401 (03): 350-353.

[8] Wei He, Pi-Cui Zhang. Soil resistivity lightning current model [J]. Applied Mechanics and Material, 2013, 403 (3): 2116-2119. 